\title{
Actigraph Calibration in Obese/Overweight and Type 2 Diabetes Mellitus Middle-Aged to Old Adult Patients
}

\author{
Vitor Pires Lopes, Pedro Magalhães, José Bragada, and Catarina Vasques
}

\begin{abstract}
Background: Several methods exist to asses and control physical intensity levels of subjects engaged in physical activities programs, accelerometry is a method that could be easily used in the field. The purposes were: to calibrate Actigraph in middle-aged to old obese/overweight and DM2 adult patients; and to determine the threshold counts for sedentary, light, moderate, and vigorous physical activity (PA). Methods: Sample comprise 26 participants (62.6 \pm 6.5 years of age) of both gender. Counts and $\mathrm{VO} 2$ were simultaneously assessed during: resting, seating, standing, walking at 2.5 $\mathrm{km} \cdot \mathrm{h}^{-1}, 5 \mathrm{~km} \cdot \mathrm{h}^{-1}$, and $6 \mathrm{~km} \cdot \mathrm{h}^{-1}$. A hierarchical linear model was used to derive a regression equation between MET and counts. Receiver operating characteristics (ROC) analysis was used to define thresholds for PA levels. Results: The regression equation was: MET = $1.388400490262+0.001312683420044$ (counts $\cdot \mathrm{min}^{-1}$ ), $r=.867$. The threshold counts for sedentary-light, light-moderate and moderate-vigorous PA were: 200, 1240, 2400 counts $\mathrm{min}^{-1}$ respectively. Conclusion: The Actigraph is a valid and useful device for the assessment of the amount of time spent in each PA intensity levels in obese/overweight and DM2 middle-aged to old adult patients.
\end{abstract}

Keywords: intervention study, exercise prescription, motion sensors

One of the most important factors in preventing and treating obesity as well as some of the morbidities associated with it, namely type 2 diabetes mellitus (DM2), is physical activity ${ }^{1}$ (PA). Regular PA is a fundamental part of lifestyle behavior in treating DM $2,{ }^{2}$ and on the primary prevention of this disease..$^{1,3,4}$ There are several

The authors are with the Dept of Sport Science, Polytechnic Institute of Bragança, Portugal. benefits of regular PA in individuals with DM2: it reduces the risk of cardiovascular disease, ${ }^{5}$ glycated hemoglobin A1c, ${ }^{2}$ blood pressure, ${ }^{3,6}$ and total abdominal fat: ${ }^{7}$ it also improves insulin sensitivity, ${ }^{7}$ glycemic control, ${ }^{6,8}$ and increases the muscle mass. ${ }^{9}$ Regular moderate-intensity PA can be used to improve metabolic risk variables such as insulin and leptin in overweight/obese postmenopausal women. ${ }^{10}$

Since PA improves energy expenditure which can cause a negative energetic imbalance, it should be considered as one of the most important factors in weight control. As a consequence, PA is important for the prevention of comorbidities associated with overweight and obesity, namely DM2.

In this context, it is important to control the exercise and PA intensity of obese and diabetic individuals that are engaged in PA programs. Among several instruments available for that purpose, accelerometers have been used as objective measurement tools, that allow the quantification of the amount of time spent in light ( $<3$ metabolic equivalents, or METs), moderate (3-5.99 METs), and vigorous ( $\geq 6$ METs) PA. This way, accelerometers should be calibrated to have population specific energy expenditure equations. ${ }^{11,12}$ This is due to the fact that PA habits change with age. On one hand, children are characterized as having short bouts of relatively intense PA interspersed with frequent rest periods. ${ }^{13,14}$ On the other hand, adults have continuous periods of PA, mostly in ambulatory activities. In older adults, the main mode of PA is walking and other ambulatory activities, and they perform significantly fewer minutes of moderate to vigorous PA than young adults. ${ }^{15}$ The measurement of lifestyle activities with accelerometers is somewhat limited. Nevertheless they measure locomotor activities quite well, ${ }^{16}$ which makes them quite suitable to measure PA in an old adult population.

There are some previous calibration studies performed on adults, although they were done with young and healthy adults. ${ }^{16-19}$ As far as we know, no calibration studies with middle-aged to old obese/overweight and DM2 adult patients were performed.

For the same physical activity effort obese/overweight subjects spend more energy than normal weight 
subjects. In fact, body weight is a major factor in rest metabolic rate and total daily energy expenditure. In addition, it seems that DM2 patients of 61 years of age have lower peak $\mathrm{VO}_{2}$ than nondiabetic patients. ${ }^{20}$ For these reasons it is important to have specific counts cutoff values for this specific group of subjects.

The purposes of this study were to calibrate Actigraph (The ActiGraph, LLC., Pensacola, FL, USA), formerly the Manufacturing Technology Incorporated Actigraph, and the Computer Science Applications accelerometer, in middle-aged to old obese/overweight and DM2 adult patients, and to determine the thresholds counts for sedentary, light, moderate, and vigorous levels of PA in ambulatory activities.

\section{Methods}

\section{Study Participants}

The participants were recruited from a population of patients with DM2, enrolled in a PA program, designed for secondary prevention and as a complementary treatment of DM2. After their assistant physician found no counter-indication for the practice of physical exercise, the patients were invited to participate in the PA program. Only the subjects with no diabetic chronic complications, who only take oral antidiabetic medication, with diagnosed diabetes for over 2 years and who were obese or overweight, were selected for the current study.

The sample for calibration (sample 1) comprised 14 middle-aged to old adults of both genders, with $63 \pm 7$ years of age. The sample for validation (sample 2) comprised 12 middle-aged to old adults of both genders, with $62.2 \pm 6$ years of age. All participants have been taking oral antidiabetic medication (metformin), but no insulin, and they did not have any chronic diabetic complications. There is the assumption that metformin could influence the $\mathrm{VO}_{2}$, however, the available research ${ }^{21-24}$ is not conclusive about this issue. All participants were white, and their characteristics are shown, by sample, in Table 1.

All the selected participants had to sign a document, to give permission to proceed with the evaluations and the study was approved by the ethical committee of the local health authority.

\section{Instrumentation}

Oxygen consumption $\left(\mathrm{VO}_{2}\right)$ and heart rate were measured using a stationary breath-by-breath metabolic unit (Cortex, Model MetaLyzer 3B, Leipzig, Germany), which was calibrated with standard gases before each test was performed. The unit includes a heart rate transmitter (Polar Electro Oy, Kempele, Finland).

Each participant wore 2 Actigraph (The ActiGraph, LLC., Pensacola, FL, USA), formerly the Manufacturing Technology Incorporated Actigraph, and the Computer Science Applications accelerometer, over their right hip, to prevent any malfunction of the PA monitor, or other mishap. The Actigraphs were the same for all participants. Both units were previously calibrated according to the manufacturer's parameters. The variation between the results obtained by both units were analyzed by means of intraclass correlation, thus result-

Table 1 Sample Characteristics

\begin{tabular}{lcc}
\hline Characteristic & $\begin{array}{c}\text { Sample 1 } \\
\text { (n=14) }\end{array}$ & $\begin{array}{c}\text { Sample 2 } \\
\text { (n = 12) }\end{array}$ \\
\hline Female (\%) & 50 & 66.7 \\
Mean age (y) & $63 \pm 7$ & $62.2 \pm 6$ \\
Education level (\%) & & 41.7 \\
$\quad$ Secondary education & 57.2 & 58.3 \\
$\quad$ Primary school & 42.8 & $162.63 \pm 3.54$ \\
Height & & $155.10 \pm 7.99$ \\
$\quad$ Male & $168.07 \pm 5.18$ & \\
$\quad$ Female & $151.49 \pm 8.54$ & $75.90 \pm 16.03$ \\
Weight & & $72.19 \pm 17.58$ \\
$\quad$ Male & $80.32 \pm 7.21$ & $29.33 \pm 4.85$ \\
$\quad$ Female & $77.05 \pm 21.03$ & 41.7 \\
Body mass index & $31.00 \pm 5.17$ & 58.3 \\
Obese (\%) & 57.1 & $7.34 \pm 1.81$ \\
Overweight (\%) & 42.9 & $148.87 \pm 35.63$ \\
HbAlc (\%) & $7.20 \pm 1.8$ & $9.25 \pm 4.47$ \\
Fasting glucose (mg/dL) & $145.43 \pm 34.30$ & $1.53 \pm 0.72$ \\
Insulin (uUI/mL) & $9.60 \pm 4.41$ & $1.59 \pm 0.71$ \\
IR (HOMA-IR) & & \\
\hline
\end{tabular}


ing in a high value $(r=.99)$. Only the data of the same unit were used for the subsequent analyzes. Activity counts were stored in 1-min time intervals.

\section{Study Design}

Since the majority of the activities proposed in the PA program involving the recruited participants had walking as the main physical activity, and since walking is the most frequent PA in this age group, ${ }^{25}$ it was decided to perform a laboratory protocol which included walking using a treadmill.

All participants were asked to present themselves at the laboratory 3 hours after their last meal. To evaluate $\mathrm{VO}_{2}$ and Actigraph counts simultaneously, each participant did the following physical activities in sequence: resting, seating, standing, walking at $2.5 \mathrm{~km} \cdot \mathrm{h}^{-1}, 5$ $\mathrm{km} \cdot \mathrm{h}^{-1}$, and $6 \mathrm{~km} \cdot \mathrm{h}^{-1}$. These activities were intended to cover rest, light, moderate, and vigorous PA intensities.

Rest $\mathrm{VO}_{2}$ was collected for $15 \mathrm{~min}$ with the participants in a lying position, in a silent and dimly lighted room. Participants were not allowed to sleep. Minutes 6 to 15 were used for data analysis. For all activities other than resting, $\mathrm{VO}_{2}$, heart rate and Actigraph counts were collected during $6 \mathrm{~min}$, and the mean value of the last 3 min was used for data analysis. During this period, the participants were considered to be at a steady-state energy expenditure since it was a submaximal physical effort. ${ }^{26}$

All participants attended, at least, 1 visit to the laboratory, before the visit to collect $\mathrm{VO}_{2}$, heart rate, and accelerometry data. In these visits, weight, height, waist and hip circumferences, as well as skinfolds (subscapular, triceps, suprailium, midthigh and medial-calf) were measured. Another purpose of these visits was to let the participants to walk on the treadmill to get used to it. The same protocol was applied to both samples.

\section{Statistical Analysis}

A hierarchical linear model was used to analyze the relationship between Actigraph counts and MET, over the walking activity intensities. This procedure allows the accounting for the dependence among repeated measures in the same subject. Restricted maximum likelihood estimates of random effects and maximum likelihood estimates of fixed effects were obtained using SPSS $15 .{ }^{27}$ Coefficients associated with quadratic and cubic trends in third polynomial model were not found to be significantly different from zero. The contribution of body mass index (BMI), waist-to-hip ratio, sum of skinfolds and gender was tested. None of them was found to contribute significantly to the fit of the model. Thus, only results from the unadjusted hierarchical linear model are reported.

Analysis for Inner Validation. The bias, which is the difference between the predicted and the corresponding actual value of MET, and the $95 \%$ limits of absolute agreement were calculated as described in Atkinson and Nevill. ${ }^{28}$

The concordance correlation coefficient $\rho_{\mathrm{c}}{ }^{29}$ which evaluates the degree to which pairs of observations fall on the $45^{\circ}$ line through the origin, was computed between the predicted and the actual values.

Threshold for Ambulatory Physical Activity Intensity Levels. Actigraph counts corresponding to the MET cut-offs for sedentary, light, moderate, and vigorous levels of PA, were calculated from the derived equation.

Receiver operating characteristics (ROC) analysis was then applied. The sensitivity [true positives/(true positives + false negatives)] and specificity [true negatives/(true negatives + false positives)] of the thresholds for counts were calculated. The cut-offs counts were selected having in consideration the highest values of sensitivity and specificity that had simultaneously the largest area under the curve. This was done using data provided by both samples.

\section{Results}

\section{Study Subjects Characteristics}

In sample 1, the body max index (BMI) was $28.4 \pm 2.1$ and $33.1 \pm 6.2$, respectively, for male and female subjects. The average waist-to-hip ratio was $0.97 \pm 0.1$ for male and $0.96 \pm 0.1$ for female. Rest metabolic rate (RMR) was $3.48 \pm 1.15$ and $2.37 \pm 0.30 \mathrm{~mL} \cdot \mathrm{kg}^{-1} \cdot \mathrm{min}^{-1}$ for men and women, respectively. In sample 2 , the BMI for males was identical to sample $1(28.5 \pm 4.9)$, but slightly lower for females $(29.7 \pm 5.4)$. The average waist-to-hip ratio for male was also identical to the sample $1(0.95 \pm 0.03)$, and again slightly lower for women $(0.88 \pm 0.02)$. Rest metabolic rate was $3.15 \pm$ 0.95 and $2.32 \pm 0.55 \mathrm{~mL} \cdot \mathrm{kg}^{-1} \cdot \mathrm{min}^{-1}$ for men and women, respectively.

The differences between samples (male and female combined) in height, weight and BMI were not statistically significant, apart from waist-to-hip ratio $\left(\mathrm{t}_{(22)}=\right.$ $2,97, P=.007)$. There were no significant differences in RMR between samples. Considering the joined samples, half of the participants were obese $(n=13)$ and the other half were overweight $(n=13)$.

The performance data obtained during the experimental protocol are presented in Table 2. In both samples, the performance indicators improved over ambulatory PA intensities. There were no significant differences between samples in any of the PA intensities in any variable. The only exception was in counts in standing activity, where sample 1 had more counts. The chosen activities provided a wide range in $\mathrm{VO}_{2}$ (3-23.9 $\mathrm{ml} \cdot \mathrm{kg}^{-1} \cdot \mathrm{min}^{-1}$ ) with a corresponding wide range in Actigraph counts $\left(2.3-5389.5\right.$ counts $\left.\mathrm{min}^{-1}\right)$. 


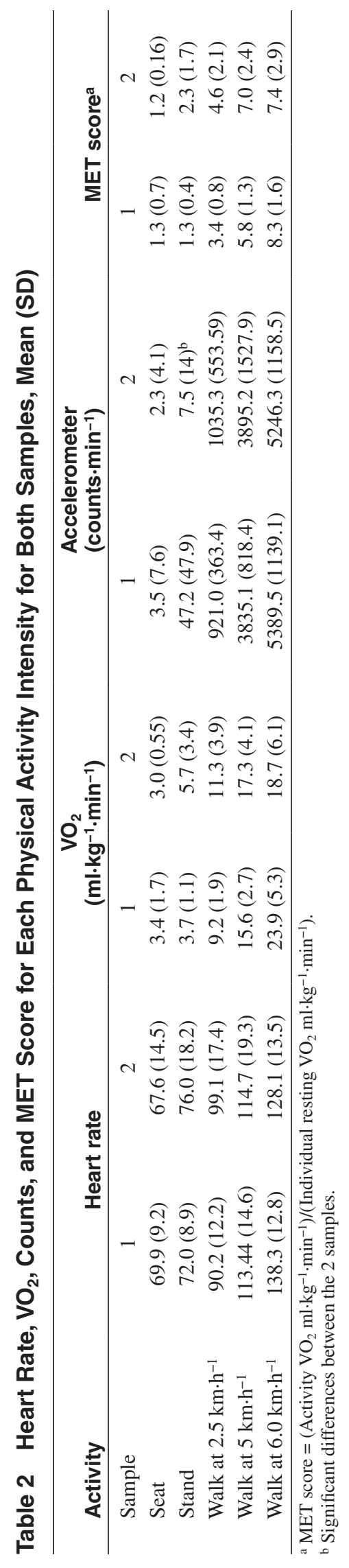




\section{Prediction Equation for MET Scores from Counts}

The data of all ambulatory PA intensities and the data of resting were used to derive the equation for MET scores. This procedure allows the anchoring of the intercept term of the equation on the low end of MET scores. Figure 1 shows the regression lines for MET scores versus Actigraph counts for each subject (thin lines) and the overall regression line (thick line). Equation 1 estimates MET scores using counts. METs were calculated as energy expenditure for each PA level divided by the subject measured rest metabolic rate.

$$
\begin{gathered}
\text { MET }=1.388400490262+0.001312683420044 \\
\left(\text { counts } \cdot \mathrm{min}^{-1}\right)[1]
\end{gathered}
$$

The measured MET scores were regressed against estimated MET scores, an $r=.867$ was obtained, so $75.2 \%$ of the variation in measured MET scores in the sample was explained by the counts.

\section{Analysis for Inner Validation}

The regression equation derived in sample 1 for prediction MET scores was used to estimate MET scores in sample 2. The predicted MET scores was significantly correlated with measured MET scores $(r=.781, P<$ $.01)$.

\section{Agreement}

The bias for sample 1 and sample 2 was $0.21 \pm 1.33$ and $-0.13 \pm 1.95$, respectively. So, for a new individual from the studied population, it would be expected (an approximate $95 \%$ probability) that the difference between the actual and predicted value should lie within the limits of agreement. Therefore, in sample 2, it would be expected that the differences between the actual and predicted

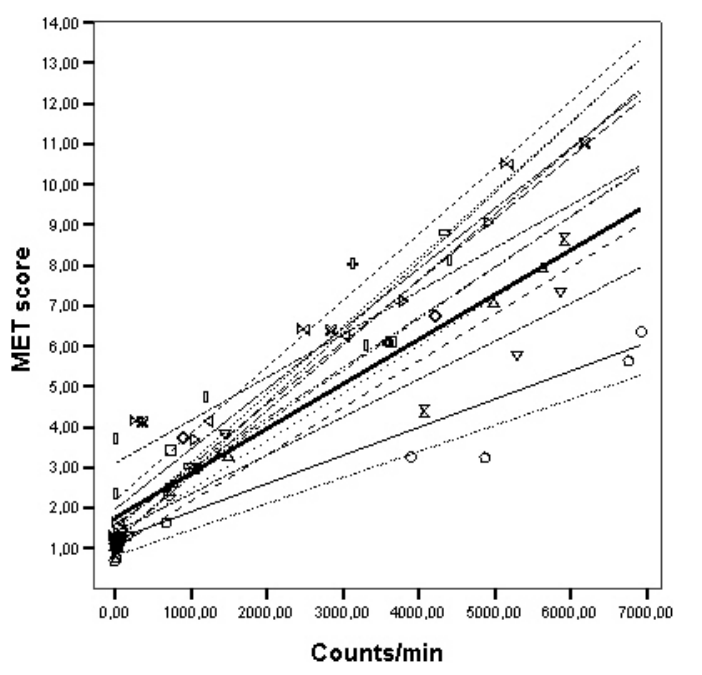

Figure 1 - Regression line for MET score versus accelerometer (Actigraph) counts (random regression line for each individual and overall regression line). value lie between -3.97 and 3.70 MET, and in sample 1 between -2.40 and 2.82 . In both cases it is an enough narrow interval for the model to be of practical use.

The value of the concordance correlation coefficient between predicted and actual values of joined samples was high $\left(\rho_{c}=0.80\right)$.

\section{Thresholds for Sedentary, Light, Moderate, and Vigorous Intensity of Ambulatory Physical Activity}

The thresholds corresponding to sedentary ( $<1.5 \mathrm{MET})$, light (1.5 to $3 \mathrm{MET})$, moderate (3-6 MET), and vigorous (6-9 MET) levels of ambulatory PA, the associated sensitivity and specificity, and the area under the curve, determined by binary classification (sedentary-light, light-moderate, moderate-vigorous) are presented in Table 3. The resulting ROC curve characterizes the performance of a binary classification by describing the trade-off between sensitivity and specificity, over an entire range of possible thresholds. Several values between 1 and 300 counts $\min ^{-1}$, were tested in the threshold for sedentary-light. The counts $\cdot \mathrm{min}^{-1}$ values of $\pm 300, \pm 1300$, and \pm 1000 given by the inverted MET equation were respectively tested in the thresholds for light-moderate, and moderate-vigorous.

The ROC analysis indicates excellent results for threshold counts for sedentary-light, light-moderate and moderate-vigorous with the area under the curve higher than 0.90 . The sensitivity was $100 \%$ for all thresholds, except for sedentary-light. Specificity was lower for moderate-vigorous $(69.74 \%)$ than in the other thresholds $(76.81 \%$ to $94 \%)$.

\section{Discussion}

The purposes of this study were to calibrate Actigraph in obese/overweight and DM2 middle-aged to old adult patients, as well as to determine the thresholds of Actigraph counts for sedentary, light, moderate, and vigorous levels of ambulatory PA.

Our data revealed that the Actigraph counts were highly correlated with MET scores, in fact, Actigraph counts accounted for $75.2 \%$ of variation in observed MET scores. The equation for MET estimation from counts, allows the use of the Compendium of Physical Activities to estimate energy expenditure over a wide range of ambulatory activities. ${ }^{30}$ The contribution of BMI, waist-to-hip ratio, sum of skinfolds and gender were found to be non significant to the fit of the model. Conversely, Freedson et $\mathrm{l}^{18}$ included BMI in the equation to predict energy expenditure in young male (24.4 \pm 4.2 years of age) and female $(22.9 \pm 3.8$ years of age $)$ adults.

It was found that 1 MET was slightly lower (3.48 \pm 1.15 and $2.37 \pm 0.30 \mathrm{~mL} \cdot \mathrm{kg}^{-1} \cdot \mathrm{min}^{-1}$ for men and women, respectively) than $3.5 \mathrm{~mL} \cdot \mathrm{kg}^{-1} \cdot \mathrm{min}^{-1}$, adopted for the adult population, so the participant's actual resting 
Table 3 Threshold Counts for Sedentary, Light, Moderate, Vigorous, and Very Vigorous Levels of Physical Activity

\begin{tabular}{lccccc}
\hline Physical activity level & MET & Counts & Sensitivity & Specificity & Area under the curve \\
\hline Sedentary & $<1.5$ & & & & \\
Light & 1.5 & 200 & 91.55 & 94.00 & 0.98 \\
Moderate & 3 & 1240 & 100 & 76.81 & 0.95 \\
Vigorous & 6 & 2400 & 100 & 69.74 & 0.94 \\
\hline
\end{tabular}

values were used to estimate MET levels. This procedure reduced the bias in the estimated energy cost of the physical activities measured in these middle-age to old adults, and are consonant with the fact that RMR decline with age. ${ }^{31,32}$

The original part of this study is that there is not, as far as we know, any study that has tried to derive an equation to MET scores through Actigraph counts, for obese/overweight and DM2 middle-aged to old adult patients. There are several equations to estimate energy expenditure through accelerometry counts for adults, but all of them are for young and healthy adults. ${ }^{18,33,34}$ To our knowledge, there is only 1 study done with a sample of older subjects which suffer from coronary artery disease..$^{35}$ In this study Actigraph was validated in a sample of 34 old adults ( $\geq 70$ years) coronary artery disease patients. They also used the walking activity in the treadmill at different speeds $\left(3.2,4.8\right.$ and $\left.6.4 \mathrm{~km} \cdot \mathrm{h}^{-1}\right)$, almost the same walking speed that was used in the current study.

The use of generalized equations in old adults is not overall recommended, since there are differences in muscle mass, gait, and other functional aspects, ${ }^{36}$ and because all of the known equations reporting MET used the value of $1 \mathrm{MET}=3.5 \mathrm{kcal} \cdot \mathrm{kg}^{-1} \cdot \mathrm{min}^{-1}$ (which is an estimate of resting energy expenditure) in their measured MET calculation. In this study the subjects had, on average, a value lower than $3.5 \mathrm{kcal} \cdot \mathrm{kg}^{-1} \cdot \mathrm{min}^{-1}$, especially women. This is another reason that justifies the use of specific calibration equations. ${ }^{11,12}$ The use of MET score based on the individual resting energy expenditure could lead to a different number of counts necessary to achieve a certain PA intensity. In the sample of this study it was obtained a $\mathrm{VO}_{2}$ resting value lower than $3.5 \mathrm{ml} \cdot \mathrm{kg}^{-1} \cdot \mathrm{min}^{-1}$. This fact had the consequence of decreasing the number of counts necessary to achieve a certain PA intensity in contrast with the MET score based on $3.5 \mathrm{ml} \cdot \mathrm{kg}^{-1} \cdot \mathrm{min}^{-1}$.

In the current study a high validity value $\left(r^{2}=.75\right)$ was obtained. The predicted energy expenditure found by Freedson et al ${ }^{18}$ in adults, while walking at different speeds on a treadmill, from Actigraph counts and BMI, obtained an $r^{2}=.82$. In a calibration study of Biotrainer and Actitrac activity monitors, Welk et al, ${ }^{34}$ found a high validation coefficients ( $r^{2}=.88$ to 0.94 ) in walking/jogging on a treadmill. In another study, Nilsson et $\mathrm{al}^{37}$ examined the effect of monitor placement (hip vs. back) and activity setting (treadmill vs. track) on the output of Actigraph and obtained high validation coefficients $\left(r^{2}\right.$
$=.85$ to 0.89 ). In the equation developed in old adult patients with coronary artery disease, Ekelund et al ${ }^{35}$ found a validation of $r^{2}=.72$. Thus, the equation obtained in the current study has a comparable validation coefficient with the previous studies. The results of inner validation also show that the equation has good indicators, which support its practical use. The limits of agreement were relatively narrow and the concordance correlation coefficient was high.

A criticism that can be made to this study is the fact that it used a laboratory protocol (walking on a treadmill) instead of free-living activities. However, the mode of PA of adults, and especially old adults, is predominantly walking. ${ }^{25,38}$ Thus, on the laboratory treadmill, the subjects performed the most frequent mode of PA in this population group.

The current study could benefit if it had embraced a larger sample, but there were some difficulties to recruit more voluntaries. The sample subjects were recruited from a PA program designed for secondary prevention and complementary treatment of DM2. All the obese/ overweight subjects that were enrolled in the program participated in the study.

Not all the validation/calibration studies calculated cut-off counts for PA intensity levels. Moreover, none of the studies derived cut-off counts for sedentary and for light PA. Based on the results of ROC analysis, namely the area under the curve and the values of sensitivity and specificity, it can be stated that the found cut-off values would be effective in characterizing PA levels. The cutoff counts value between sedentary and light PA was 200 counts, which represent the activity with minimal body movement in a sitting or standing position. For Actigraph activity monitor in children (6 to 16 years old) Puyau et al ${ }^{39}$ found a higher value (800 counts), and Treuth et $\mathrm{al}^{40}$ observed a cut-off count value of 100 in adolescent girls.

Between light and moderate PA there was a cut-off of 1240 counts. In other studies, ${ }^{17-19}$ the values vary between 1952 and 2260. These values are slightly higher than the value found in the current study. However, the samples of those studies were, as previously explained, young and healthy adults. The cut-off count value between moderate and vigorous PA levels were 2400 counts, again a value below the cut-off values found in the literature, ${ }^{17-19}$ which vary between 5725 and 6893.4 counts. In the sample of the current study the resulted cut-off points were nearly 1000 Actigraph counts less than those in the literature. These differences could be 
explained by the particular characteristics of the sample used, namely the older age, the obesity/overweight and the disease conditions. Actually, it was found ${ }^{20}$ that peak $\mathrm{VO}_{2}$ was substantially lower in diabetic than in nondiabetic patients with 61 years of age. The combination of these factors may, therefore, lead to an inferior level of physical fitness. With smaller levels of physical fitness the subjects of the sample of this study reach a certain PA intensity with lower speed of walking than the younger and fitter subjects, and as a consequence, a inferior number of counts. For instance Freedson et al ${ }^{18}$ for $4.8 \mathrm{~km} \cdot \mathrm{h}^{-1}$ walking speed found $3.7 \pm 0.3$ METs, and Hendelman et al ${ }^{19}$ for a similar speed $\left(4.75 \mathrm{~km} \cdot \mathrm{h}^{-1}\right)$ found a similar PA intensity ( $3.33 \pm 0.76$ METs). In the current study for comparable walking speed $\left(5 \mathrm{~km} \cdot \mathrm{h}^{-1}\right)$ a PA intensity of $5.8 \pm 1.3$ and $7.0 \pm 2.4$ METs for sample 1 and sample 2 was obtained, respectively. The overweight/obesity by itself is a condition that can influence the number of counts in an ambulatory activity, since for the same activity a person with more weight spends more energy than others with less weight. The other reason is the use of MET score based on the individual resting energy expenditure. In fact, as it was stated above, for the same PA intensity, there will be less counts with the MET score based on the individual resting energy expenditure than with the MET score based on $3.5 \mathrm{~mL} / \mathrm{kg} / \mathrm{min}$, because the sample of this study had lower resting $\mathrm{VO}_{2}$ than $3.5 \mathrm{ml} \cdot \mathrm{kg}^{-1} \cdot \mathrm{min}^{-1}$.

The limitation of this study lays in the fact that only run and walk were used as mode of PA. Actually, the number of counts measured might depend on the mode of PA. ${ }^{16}$ This fact limits the use of the obtained regression equation and the thresholds counts of PA levels to ambulatory PA.

In conclusion, the validation of the Actigraph against MET scores and its calibration for sedentary, light, moderate, and vigorous ambulatory PA thresholds counts certifies this monitor as a valid, useful device for the assessment of the amount of time spent in several PA intensities in middle-aged to old obese and DM2 adult patients.

\section{References}

1. Tuomilehto J, Lindstrom J, Eriksson JG, Valle TT, Hamalainen $\mathrm{H}$, et al. Prevention of type 2 diabetes mellitus by changes in lifestyle among subjects with impaired glucose tolerance. N Engl J Med. 2001;344(18):1343-1350.

2. Boule NG, Haddad E, Kenny GP, Wells GA, Sigal RJ. Effects of exercise on glycemic control and body mass in type 2 diabetes mellitus: a meta-analysis of controlled clinical trials. JAMA. 2001;286(10):1218-1227.

3. Eriksson KF, Lindgärde F. Prevention of Type 2 (noninsulin-dependent) diabetes mellitus by diet and physical exercise The 6-year Malmö feasibility study. Diabetologia. 1991;34(12):891-898.

4. Ronnemaa T, Koskenvuo M, Marniemi J, et al. Glucose Metabolism in Identical Twins Discordant for Obesity.
The Critical Role of Visceral Fat. J Clin Endocrinol Metab. 1997;82(2):383-387.

5. Tanasescu M, Leitzmann MF, Rimm EB, Hu FB. Physical activity in relation to cardiovascular disease and total mortality among men with type 2 diabetes. Circulation. 2003;107(19):2435-2439.

6. Agurs-Collins TD, Kumanyika SK, Ten Have TR, Adams-Campbell LL. A randomized controlled trial of weight reduction and exercise for diabetes management in older African-American subjects. Diabetes Care. 1997;20(10):1503-1511.

7. Stewart KJ. Exercise training and the cardiovascular consequences of type 2 diabetes and hypertension: plausible mechanisms for improving cardiovascular health. JAMA. 2002;288(13):1622-1631.

8. Dunstan DW, Daly RM, Owen N, et al. High-intensity resistance training improves glycemic control in older patients with type 2 diabetes. Diabetes Care. 2002;25(10):1729-1736 [Original Article].

9. Lim J, Kang H, Stewart K. Type 2 diabetes in Singapore: the role of exercise training for its prevention and management. Singapore Med J. 2004;45(2):62-68.

10. Frank LL, Sorensen BE, Yasui Y, Tworoger SS, Schwartz RS, et al. Effects of exercise on metabolic risk variables in overweight postmenopausal women: a randomized clinical trial. Obes Res. 2005;13(3):615-625.

11. Welk GJ. Principles of design and analyses for the calibration of accelerometry-based activity monitors. Med Sci Sports Exerc. 2005;37(12):S501-S11.

12. Freedson P, Pober D, Janz KF. Calibration of accelerometer output for children. Med Sci Sports Exerc. 2005;37(12):S523.

13. Bailey RC, Olson J, Pepper SL, Porszasz J, Barstow TJ, Cooper DM. The level and tempo of children's physical activities: an observation study. Med Sci Sports Exerc. 1995;27(7):1033-1041.

14. Lopes VP, Vasques CMS, Pereira MBFLO. R MJA, Malina RM. Physical activity patterns during school recess. a study in children 6 to 10 years old. The Int Electron J Health Educ. 2006;9:192-201.

15. Davis M, Fox K. Physical activity patterns assessed by accelerometry in older people. Eur J Appl Physiol. 2007;100(5):581-589.

16. Crouter SE, Clowers KG, Bassett DR, Jr. A novel method for using accelerometer data to predict energy expenditure. J Appl Physiol. 2006;100(4):1324-1331.

17. Yngve A, Nilsson A, Sjöström M, Ekelund U. Effect of monitor placement and of activity setting on the MTI accelerometer output. Med Sci Sports Exerc. 2003;35(2):320-326.

18. Freedson P, Melanson E, Sirard J. Calibration of the Computer Science and Applications, Inc. accelerometer. Med Sci Sports Exerc. 1998;30(5):777-781.

19. Hendelman D, Miller K, Baggett C, Debold E, Freedson P. Validity of accelerometry for the assessment of moderate intensity physical activity in the field. Med Sci Sports Exerc. 2000;32(9, supplement):S442-S9.

20. Tibb AS, Ennezat PV, Chen JA, Haider A, Gundewar S, Cotarlan V, Aggarwal VS, Talreja A, Le Jemtel TH. Dia- 
betes lowers aerobic capacity in heart failure. $J$ Am Coll Cardiol. 2005;46(5):930-931.

21. Brunmair B, Staniek K, Gras F, Scharf N, Althaym A, Clara R, Roden M, Gnaiger E, Nohl H, Waldhausl W, Furnsinn C. Thiazolidinediones, like Metformin, inhibit respiratory complex i: a common mechanism contributing to their antidiabetic actions? Diabetes. 2004;53(4):10521059.

22. Cunha MR, Silva MER, Watanabe E, et al. Metabolic responses to the maximal exercise test in patients with type 2 diabetes treated with metformin. Comparison with healthy controls. Diabetes Res Clin Pract. 2000;50(Supplement 1):52.

23. Braun B, Eze P, Stephens BR, Hagobian TA, et al. Impact of metformin on peak aerobic capacity. Appl Physiol Nutr Metab. 2008;33:61-67.

24. Guigas B, Detaille D, Chauvin C. Batandier Cc, De Oliveira Fdr, Fontaine E, Leverve X. Metformin inhibits mitochondrial permeability transition and cell death: a pharmacological in vitro study. Biochem $J$. 2004;382(3):877-884.

25. Owen N, Humpel N, Leslie E, Bauman A, Sallis JF. Understanding environmental influences on walking. $A m$ J Prev Med. 2004;27(1):67-76.

26. Robergs RA, Roberts SO. Ergometry and calorimetry in exercise physiology, exercise, performance and clinical applications. St. Louis, Missouri: Mosby-Year Book, Inc; 1997.

27. SPSS. SPSS for Windows. 13 ed. Chicago: SPSS; 2004.

28. Atkinson G, Nevill AM. Statistical methods for assessing measurement error (reliability) in variables relevant to sports medicine. Sports Med. 1998;26(4):217-238.

29. Lin LI. A note on the concordance correlation coefficient. Biometrics. 2000;56(1):324-325.

30. Ainsworth BE, Haskell WL, Whitt MC, et al. Compendium of physical activities: an update of activities codes and MET intensities. Med Sci Sports Exerc. 2000;32(9, supplement):S498-S504.

31. Poehlman ET, Goran MI, Gardner AW, Ades PA, Arciero PJ, Katzman-Rooks SM, Montgomery SM, Toth MJ,
Sutherland PT. Determinants of decline in resting metabolic rate in aging females. Am J Physiol Endocrinol Metab. 1993;264(3):E450-455.

32. Vaughan L, Zurlo F, Ravussin E. Aging and energy expenditure. Am J Clin Nutr. 1991;53(4):821-825.

33. Nichols JFM, Cindy G. Sarkin, Julie A.; Sallis, James F.; Calfas, Karen J. Validity, reliability, and calibration of the Tritrac accelerometer as a measure of physical activity. Med Sci Sports Exerc. 1999;31(6):908-912.

34. Welk GJ, Almeida J, Morss G. Laboratory Calibration and Validation of the Biotrainer and Actitrac Activity Monitors. Med Sci Sports Exerc. 2003;35(6):1057-1064.

35. Ekelund U, Tingstrom P, Kamwendo K, Krantz M, Nylander E, Sjostrom M. The validity of the computer science and applications activity monitor for use in coronary artery disease patients during level walking. Clin Physiol Funct Imaging. 2002;22(4):248-253.

36. Reilly MA, Spirduso WW. Age-related differences in response programming. Res $Q$ Exerc Sport. 1991;62(2):178-186.

37. Nilsson A, Ekelund U, Yngve A, Sjöström M. Assessing physical activity among children with accelerometers using different time sampling intervals and placements. Pediatr Exerc Sci. 2002;14(1):87-96.

38. Kavanagh AM, Goller JL, King T, Jolley D, Crawford D, Turrell G. Urban area disadvantage and physical activity: a multilevel study in Melbourne, Australia. J Epidemiol Community Health. 2005;59(11):934-940.

39. Puyau MR, Adolph AL, Vohra FA, Butte NF. Validation and Calibration of Physical Activity Monitors in Children. Obes Res. 2002;10(3):150-157.

40. Treuth MS, Schmitz K, Catellier DJ, et al. Defining accelerometer thresholds for activity intensities in adolescent girls. Med Sci Sports Exerc. 2004;36(7):1259-1266. 